\title{
焙烧气氛对 $\mathrm{LiNi}_{0.5} \mathrm{Mn}_{0.5} \mathrm{O}_{2}$ 中 $\mathrm{Li} / \mathrm{Ni}$ 混排及电化学性能的影响
}

\author{
王晓亚 程 前 黄 桃 余爱水 ${ }^{*}$ \\ (复旦大学化学系, 新能源研究院, 上海催化材料功能研究实验室, 上海 200438)
}

\begin{abstract}
摘要: 用固相法分别在氧气和空气气氛下合成了层状锂离子电池正极材料 $\mathrm{LiNi}_{0.5} \mathrm{Mn}_{0.5} \mathrm{O}_{2}$, 采用 $\mathrm{X}$ 射线衍射 $(X R D)$ 、扫描电镜(SEM)、透射电镜(TEM)、电化学阻抗谱(EIS)及充放电性能测试对其结构、形貌和电化学性质 进行表征, 用 Rietveld 精修计算晶体结构中的 Li/Ni混排率, 研究了混排率与电化学性能的关系. 结果显示, 在 不同的焙烧气氛下均能合成出纯相和结晶性良好的 $\mathrm{LiNi}_{0.5} \mathrm{Mn}_{0.5} \mathrm{O}_{2}$, 但两种材料在电化学性能上存在一定的差 异. 氧气气氛下焙烧合成的材料在首次放电容量, 循环稳定性方面均优于空气气氛下合成的材料. 在 $0.1 C$ 充放 电条件下氧气气氛下焙烧得到的 $\mathrm{LiNi}_{0.5} \mathrm{Mn}_{0.5} \mathrm{O}_{2}$ 材料首次放电容量达到 $178 \mathrm{mAh} \cdot \mathrm{g}^{-1}$, 充放电循环 50 圈后容量 为 $165 \mathrm{mAh} \cdot \mathrm{g}^{-1}$, 容量保持率为 $92.7 \%$; 而在空气气氛下焙烧得到的 $\mathrm{LiNi}_{0.5} \mathrm{Mn}_{0.5} \mathrm{O}_{2}$ 材料首次放电容量为 164 $\mathrm{mAh} \cdot \mathrm{g}^{-1}$, 充放电循环 50 圈后容量为 $137 \mathrm{mAh} \cdot \mathrm{g}^{-1}$, 容量保持率为 $83.5 \%$. 氧气气氛下合成的材料具有较优的 电化学性能可归因于氧气气氛下焙烧合成的 $\mathrm{LiNi}_{0.5} \mathrm{Mn}_{0.5} \mathrm{O}_{2}$ 具有较小的 $\mathrm{Li} / \mathrm{Ni}$ 混排率.
\end{abstract}

关键词: 锂离子电池; 正极材料; $\mathrm{LiNi}_{0.5} \mathrm{Mn}_{0.5} \mathrm{O}_{2} ; \mathrm{Li} / \mathrm{Ni}$ 混排; 焙烧气氛

中图分类号: 0646

\section{Effect of Calcination Atmosphere on Li/Ni Disorder and Electrochemical Performance of Layered LiNi $\mathrm{Li}_{0.5} \mathrm{Mn}_{0.5} \mathrm{O}_{2}$}

\author{
WANG Xiao-Ya CHENG Qian HUANG Tao YU Ai-Shui* \\ (Department of Chemistry, Shanghai Key Laboratory of Molecular Catalysis and Innovative Materials, Institute of New Energy, \\ Fudan University, Shanghai 200438, P. R. China)
}

\begin{abstract}
Layered $\mathrm{LiNi}_{0.5} \mathrm{Mn}_{0.5} \mathrm{O}_{2}$ was synthesized by a solid state reaction method under air or oxygen atmosphere. The obtained materials were characterized by X-ray diffraction (XRD), scanning electron microscopy (SEM), transmission electron microscopy (TEM), electrochemical impedance spectroscopy (EIS), and charge-discharge tests. The results show that the $\mathrm{LiNi}_{0.5} \mathrm{Mn}_{0.5} \mathrm{O}_{2}$ synthesized by the solid state reaction method under both air and oxygen atmospheres give a pure phase and good crystallinity, however, their electrochemical performance differs. The material synthesized under oxygen gives better electrochemical performance including a higher first discharge capacity and better cycle stability. At a rate of $0.1 \mathrm{C}$ the first discharge capacity of the material synthesized under oxygen was found to be $178 \mathrm{mAh}$. $\mathrm{g}^{-1}$. After 50 charge and discharge cycles the discharge capacity was still $165 \mathrm{mAh} \cdot \mathrm{g}^{-1}$ giving a capacity retention rate of $92.7 \%$. For the material synthesized under air, the first discharge capacity at a rate of $0.1 \mathrm{C}$ was found to be $164 \mathrm{mAh} \cdot \mathrm{g}^{-1}$. After 50 charge and discharge cycles, the discharge capacity was 137 $\mathrm{mAh} \cdot \mathrm{g}^{-1}$ giving a capacity retention rate of $83.5 \%$. The reason for the material synthesized under oxygen having better electrochemical performance than the material synthesized under air is due to the oxygen atmosphere suppressing the $\mathrm{Li} / \mathrm{Ni}$ exchange ratio in $\mathrm{LiNi}_{0.5} \mathrm{Mn}_{0.5} \mathrm{O}_{2}$.
\end{abstract}

Received: October 23, 2010; Revised: December 14, 2010; Published on Web: January 13, 2011.

"Corresponding author. Email: asyu@fudan.edu.cn; Tel/Fax: +86-21-51630320.

The project was supported by the National Key Basic Research Program of China (973) (2009CB220100), National High Technology Research and Development Program of China (863) (2009AA033701), and Science \& Technology Commission of Shanghai Municipality, China (08DZ2270500). 国家重点基础研究发展规划(973) (2009CB220100), 国家高技术研究发展计划(863) (2009AA033701)及上海催化材料功能研究实验室 (08DZ2270500)资助项目

(C) Editorial office of Acta Physico-Chimica Sinica 
Key Words: Lithium ion battery; Cathode material, $\quad \mathrm{LiNi}_{0.5} \mathrm{Mn}_{0.5} \mathrm{O}_{2} ; \quad \mathrm{Li} / \mathrm{Ni}$ exchange; Calcination atmosphere

\section{1 引言}

随着社会的发展对能源的需求量越来越高, 传 统能源储量日益减少以及人们对传统能源带来的 环境问题的关注, 高能量可反复充放的锂离子电池 广泛受到关注并在现在的生产生活中发挥着越来 越重要的作用. 在锂电池的组成部分中, 正极材料 是制约锂离子电池发展的重要因素. 现有商业化的 锂离子电极正极材料主要是 $\mathrm{LiCoO}_{2}$, 但是钴资源匮 乏, 价格昂贵, 热力学稳定性差, 会造成环境污染, 因此开发可代替的正极材料具有重要意义和实际 应用前景. ${ }^{1}$ 层状的 $\mathrm{LiNi}_{0.5} \mathrm{Mn}_{0.5} \mathrm{O}_{2}$ 具有价格低廉, 理论 容量高, 循环性能良好和优异的热力学稳定性等优 点而受到广泛的关注, 有望成为商业化的 $\mathrm{LiCoO}_{2}$ 正 极材料. ${ }^{2}$ 现有的合成方法主要有固相法, 共沉淀法, 溶胶-凝胶法和离子交换法. ${ }^{2-5}$ 众所周知, 合成条件 对 $\mathrm{LiNi}_{0.5} \mathrm{Mn}_{0.5} \mathrm{O}_{2}$ 的晶体结构, ${ }^{6-10}$ 形貌, ${ }^{4,11,12}$ 结晶性和 电化学性能 ${ }^{13}$ 有重要影响. 例如, 2001 年 Ohzuku 和 Makimura $^{2}$ 首次用固相法合成的材料经过 30 圈充放 电循环后, 材料的放电容量仍然优于 $\mathrm{LiCoO}_{2}$, 达到 $150 \mathrm{mAh} \cdot \mathrm{g}^{-1} .2005$ 年, Myung 等 ${ }^{3}$ 用共沉淀法合成 了层状的 $\mathrm{LiNi}_{0.5} \mathrm{Mn}_{0.5} \mathrm{O}_{2}$, 该材料在 2.8-4.4 V 的充放 电电位区间内放电容量达到 $163 \mathrm{mAh} \cdot \mathrm{g}^{-1} .2006$ 年 Kang 等 ${ }^{5}$ 用离子交换法合成的 $\mathrm{LiNi}_{0.5} \mathrm{Mn}_{0.5} \mathrm{O}_{2}$ 材料在 $3.0-4.6 \mathrm{~V}$ 的放电区间内容量大于 $230 \mathrm{mAh} \cdot \mathrm{g}^{-1}$. 不 同合成方法或条件在合成 $\mathrm{LiNi}_{0.5} \mathrm{Mn}_{0.5} \mathrm{O}_{2}$ 材料的过程 中会导致材料具有不同的 $\mathrm{Li} / \mathrm{Ni}$ 混排率, 而层状 $\mathrm{LiNi}_{0.5} \mathrm{Mn}_{0.5} \mathrm{O}_{2}$ 正极材料中的 $\mathrm{Li} / \mathrm{Ni}$ 混排率是影响材

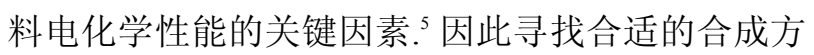
法, 控制适宜的合成条件对材料的电化学性能是至 关重要的.

本文采用固相法分别在氧气和空气两种焙烧 气氛下合成层状的 $\mathrm{LiNi}_{0.5} \mathrm{Mn}_{0.5} \mathrm{O}_{2}$ 正极材料. 采用 XRD、SEM、TEM等方法表征材料, 测试材料的电化 学性能, 并探讨焙烧气氛对晶体结构及材料电化学 性能的影响.

\section{2 实 验}

\section{$2.1 \mathrm{LiNi}_{0.5} \mathrm{Mn}_{0.5} \mathrm{O}_{2}$ 制备}

按一定的化学计量比称取 $\mathrm{Mn}\left(\mathrm{CH}_{3} \mathrm{COO}\right)_{2}$. $4 \mathrm{H}_{2} \mathrm{O}$ (国药集团化学试剂有限公司, 纯度 $>99 \%$ ),
$\mathrm{Ni}\left(\mathrm{CH}_{3} \mathrm{COO}\right)_{2} \cdot 4 \mathrm{H}_{2} \mathrm{O}$ (国药集团化学试剂有限公司, 纯度 $>98 \%$ ) 和 $\mathrm{C}_{2} \mathrm{H}_{2} \mathrm{O}_{4} \cdot 2 \mathrm{H}_{2} \mathrm{O}$ (上海强顺化学试剂有限 公司, 纯度 $>99.5 \%$ ) (摩尔比为 $1: 1: 2$ ) 放入研钵中混 合均匀研磨 $1 \mathrm{~h}$ 而后加入 $\mathrm{Li}(\mathrm{OH}) \cdot \mathrm{H}_{2} \mathrm{O}$ (国药集团化 学试剂有限公司, 纯度 $>95 \%$ ) 混合均匀研磨 $1 \mathrm{~h}$, 压 片在氧气气氛下以 $2.5^{\circ} \mathrm{C} \cdot \mathrm{min}^{-1}$ 升温速率升到 500 ${ }^{\circ} \mathrm{C}$ 并保持 $10 \mathrm{~h}$, 缓慢冷却到室温后充分研磨. 压片 后在 $800{ }^{\circ} \mathrm{C}$ 氧气气氛条件下再次焙烧 $10 \mathrm{~h}$, 升温速 率为 $5^{\circ} \mathrm{C} \cdot \mathrm{min}^{-1}$, 自然冷却到室温. 用相同的方法在 空气气氛下制备 $\mathrm{LiNi}_{0.5} \mathrm{Mn}_{0.5} \mathrm{O}_{2}$ 材料作为对比.

\section{2 材料表征与测试}

试样的晶体结构由 Bruker D8 型多晶 X 射线衍 射仪(德国布鲁克公司)进行分析, 使用 $\mathrm{Cu}$ 靶 $K_{a}$ 射线 $(\lambda=0.15406 \mathrm{~nm}), \mathrm{Ni}$ 滤波片, 扫描范围为 $10^{\circ}-90^{\circ}$, 扫 描速率为 $2\left(^{\circ}\right) \cdot \mathrm{min}^{-1}$. 用 Accelrys MS Modeling 3.0.1 软件对材料的晶胞参数和材料中的 $\mathrm{Li} / \mathrm{Ni}$ 混排率进 行精修拟合计算. 采用扫描电子显微镜(SEM, JMS 6390 日本)和透射电子显微镜(TEM)观察材料的形 貌.

\section{3 电化学测试与表征}

将质量比为 70:20:10 的 $\mathrm{LiNi}_{0.5} \mathrm{Mn}_{0.5} \mathrm{O}_{2}$ 正极材 料、导电碳黑 Super P 和聚四氟乙烯(PTFE)混合均匀 后擀膜, 切成直径为 $10 \mathrm{~mm}$ 的圆形极片. 本文中设 定 $1 C=140 \mathrm{mAh} \cdot \mathrm{g}^{-1}$, 活性物质在极片上为 $0.0127 \mathrm{~g}$. $\mathrm{cm}^{-2}$. 在压片机上用 $3 \mathrm{MPa}$ 的压力压在 $\mathrm{Al}$ 网上, 真空 干燥 $20 \mathrm{~h}$ 后将其移至氩气气氛的手套箱内, 以其为 正极, 锂片为负极, $1 \mathrm{~mol} \cdot \mathrm{L}^{-1} \mathrm{LiPF}_{6}$ 溶解于碳酸乙烯 酯/碳酸二甲酯(体积比为 1:1) 为电解液, 正、负极间 采用 Celgard 2300 聚丙烯膜组装成型号为 CR2016 的纽扣电池. 用武汉金诺电子有限公司生产的蓝电 电池测试系统进行充放电测试, 充放电电压区间为 2.5-4.3 V. 电化学交流阻抗谱实验在上海辰华仪器 有限公司生产的 CHI660A 型电化学工作站上进行, 测试频率范围为 $0.01 \mathrm{~Hz}-100 \mathrm{kHz}$, 交流信号振幅为 $5 \mathrm{mV}$. 所有的测试均在室温条件下进行.

\section{3 结果与讨论}

\section{1 材料结构与形貌表征}

图 1 是不同焙烧气氛下合成的 $\mathrm{LiNi}_{0.5} \mathrm{Mn}_{0.5} \mathrm{O}_{2}$ 的 XRD 谱图. 从图中可以看出, 两种焙烧气氛下得到 
的材料的衍射峰尖锐且对称, 衍射特征峰的位置与 标准的 $\alpha-\mathrm{NaFeO}_{2}$ 层状结构完全对应, 属于 $R \overline{3} m$ 空间 群, 未观察到杂质峰. 说明所合成的材料均为纯相的 $\mathrm{LiNi}_{0.5} \mathrm{Mn}_{0.5} \mathrm{O}_{2}$. 虽然两种材料的 XRD 谱图中没有杂 质峰出现, 但是从 XRD 图中可以观察到氧气气氛下 得到的产物 $\mathrm{LiNi}_{0.5} \mathrm{Mn}_{0.5} \mathrm{O}_{2}$ 的(003) 晶面与(104)晶面 的强度比 $I_{(003)} / I_{(104)}$ 的比值明显大于空气气氛下得到 的样品. $I_{(003)} / I_{(104)}$ 的比值与晶体结构材料中 $\mathrm{Li}$ 和 $\mathrm{Ni}$, $\mathrm{Mn}$ 过渡金属在相对应的空间内的排列有序度有 关, 比值越大, 阳离子混排效应越小, 有序程度越 高. ${ }^{14-16}$ 这说明氧气气氛下得到的 $\mathrm{LiNi}_{0.5} \mathrm{Mn}_{0.5} \mathrm{O}_{2}$ 晶体 结构中的有序度要高于空气气氛下合成的材料. 除 此之外, 在氧气气氛下合成的材料的 XRD 图中, (006)/(102)和(108)/(110)衍射峰发生分裂, 而在空气 气氛下合成的材料(006)/(102)和(108)/(110)衍射峰 重叠. 峰(006)/(102)和(108)/(110)的分裂说明材料形 成了有序的晶体结构和良好的结晶性, ${ }^{2}$ 由此得知, 氧气气氛下合成的 $\mathrm{LiNi}_{0.5} \mathrm{Mn}_{0.5} \mathrm{O}_{2}$ 材料的晶体结构有 序度和结晶性优于空气气氛下合成的样品.

由于 $\mathrm{Li}^{+} / \mathrm{Ni}^{+}$离子半径非常接近, 所以合成的 $\mathrm{LiNi}_{0.5} \mathrm{Mn}_{0.5} \mathrm{O}_{2}$ 材料会不可避免地在层状结构中出现 阳离子的混排. ${ }^{17-22}$ 为进一步确定材料中的 $\mathrm{Li} / \mathrm{Ni}$ 混 排率, 对 XRD 谱图进行 Rietveld 精修拟合计算. 在精 修拟合计算中参数 $R_{\text {wp }}$ (精修拟合计算中的 $R$ 因子轮 廓权重)和 $R_{\mathrm{p}}$ (精修拟合计算中的 $R$ 因子轮廓)值小于 $7 \%$ 时, 则表明精修结果准确可靠. 在空气, 氧气气氛 下合成 $\mathrm{LiNi}_{0.5} \mathrm{Mn}_{0.5} \mathrm{O}_{2}$ 的精修的结构如图 2 所示. 经计 算, 氧气气氛下焙烧的样品的 $\mathrm{Li} / \mathrm{Ni}$ 混排率为 $7.3 \%$ $\left(R_{\mathrm{wp}}=4.43 \%, R_{\mathrm{p}}=3.41 \%\right)$, 空气气氛下合成的样品 $\mathrm{Li} /$ $\mathrm{Ni}$ 混排率为 $8.5 \%\left(R_{\mathrm{wp}}=2.88 \%, R_{\mathrm{p}}=2.17 \%\right)$; 而且两者

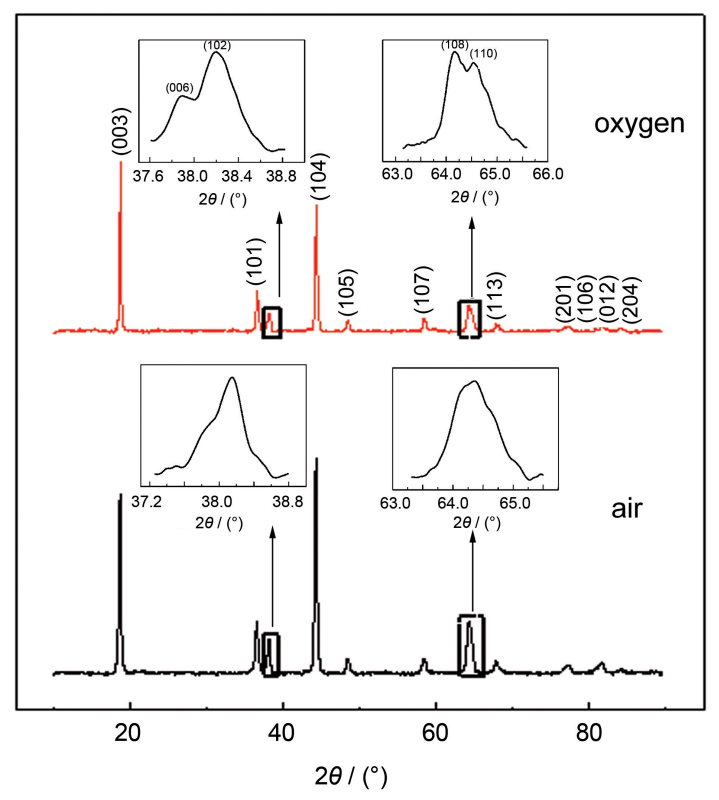

图 1 不同焙烧气氛下合成的 $\mathrm{LiNi}_{0.5} \mathrm{Mn}_{0.5} \mathrm{O}_{2}$ 的 XRD 谱图 Fig.1 XRD patterns of $\mathrm{LiNi}_{0.5} \mathrm{Mn}_{0.5} \mathrm{O}_{2}$ synthesized under different calcination atmospheres

的 $R_{\mathrm{wp}}$ 和 $R_{\mathrm{p}}$ 值均小于 $7 \%$.

图 3 给出不同焙烧气氛下合成的 $\mathrm{LiNi}_{0.5} \mathrm{Mn}_{0.5} \mathrm{O}_{2}$ 的 SEM 图. 从图 3 可以看出, 两种气氛下焙烧得到 的 $\mathrm{LiNi}_{0.5} \mathrm{Mn}_{0.5} \mathrm{O}_{2}$ 均为球状的小颗粒. 需要指出的是 在空气气氛下合成的材料表面呈棉絮状且团聚现象 较为明显, 颗粒粒径在 100-150 nm 左右, 而氧气气 氛下合成的材料颗粒相对分散, 颗粒粒径在 50-500 nm 左右. 图 4给出不同焙烧气氛下合成的 $\mathrm{LiNi}_{0.5} \mathrm{Mn}_{0.5} \mathrm{O}_{2}$ 的 TEM 图. 从图 4 可以看出, 较大的颗 粒是由一些小颗粒组成的. 单个颗粒大小范围在 50-150 nm 左右, 这些小颗粒团聚成大颗粒的大小 为 $300-500 \mathrm{~nm}$.

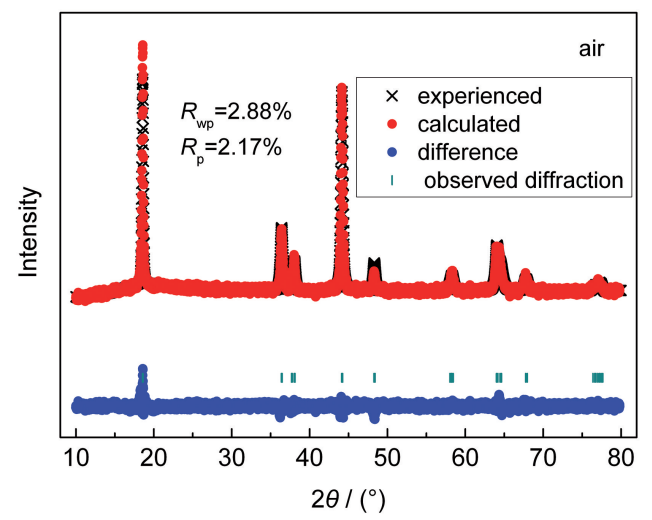

图 2 不同焙烧气氛下合成 $\mathrm{LiNi}_{0.5} \mathrm{Mn}_{0.5} \mathrm{O}_{2}$ 的精修图

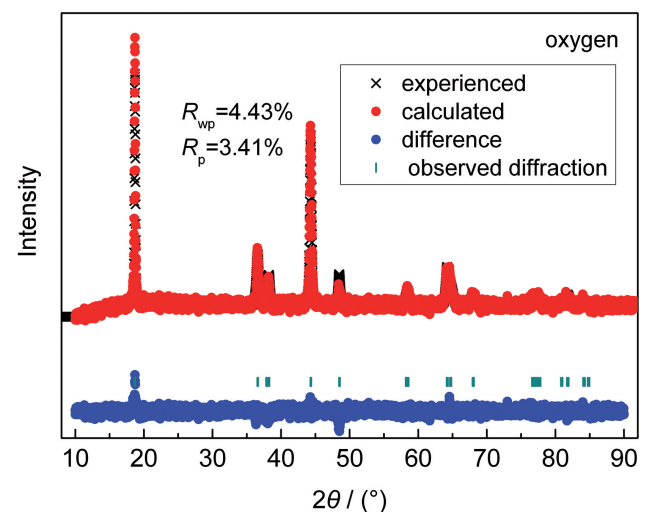

Fig.2 Rietveld refinement of $\mathrm{LiNi}_{0.5} \mathrm{Mn}_{0.5} \mathrm{O}_{2}$ synthesized under different calcination atmospheres $R_{\text {wp }}$ is the weighted profile $R$-factor, $R_{\mathrm{p}}$ is the profile $R$-factor. 

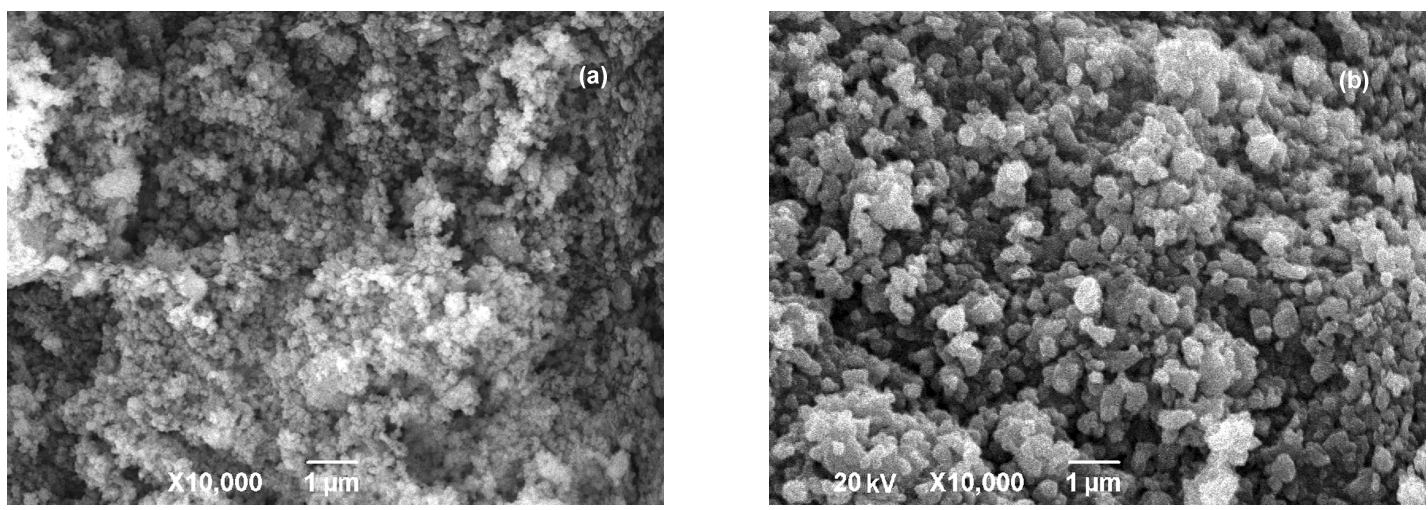

图 3 不同焙烧气氛下合成的 $\mathrm{LiNi}_{0.5} \mathrm{Mn}_{0.5} \mathrm{O}_{2}$ 的 SEM 谱图

Fig.3 SEM images of $\operatorname{LiNi}_{0.5} \mathrm{Mn}_{0.5} \mathrm{O}_{2}$ synthesized under different calcination atmospheres

(a) air, (b) oxygen
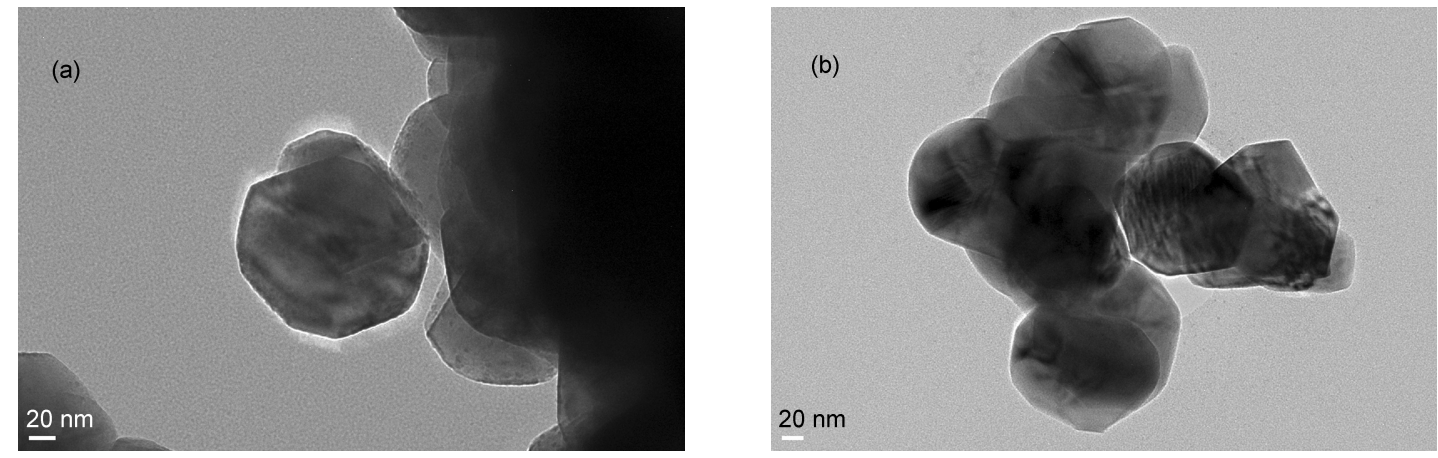

图 4 不同焙烧气氛下合成的 $\mathrm{LiNi}_{0.5} \mathrm{Mn}_{0.5} \mathrm{O}_{2}$ 的 TEM 谱图

Fig.4 TEM images of $\mathrm{LiNi}_{0.5} \mathrm{Mn}_{0.5} \mathrm{O}_{2}$ snythesized under different calcination atmospheres

(a) air, (b) oxygen

\section{2 电化学性能测试与分析}

图 5 是不同焙烧气氛下合成的 $\mathrm{LiNi}_{0.5} \mathrm{Mn}_{0.5} \mathrm{O}_{2}$ 的 首次放电图. 从图中可以看出, 在氧气气氛下焙烧合 成的 $\mathrm{LiNi}_{0.5} \mathrm{Mn}_{0.5} \mathrm{O}_{2}$ 首次放电容量达到 $178 \mathrm{mAh} \cdot \mathrm{g}^{-1}$, 优于空气焙烧气氛下的 $164 \mathrm{mAh} \cdot \mathrm{g}^{-1}$. 这可能是因为 在氧气气氛下合成的 $\mathrm{LiNi}_{0.5} \mathrm{Mn}_{0.5} \mathrm{O}_{2}$ 具有更好的结晶

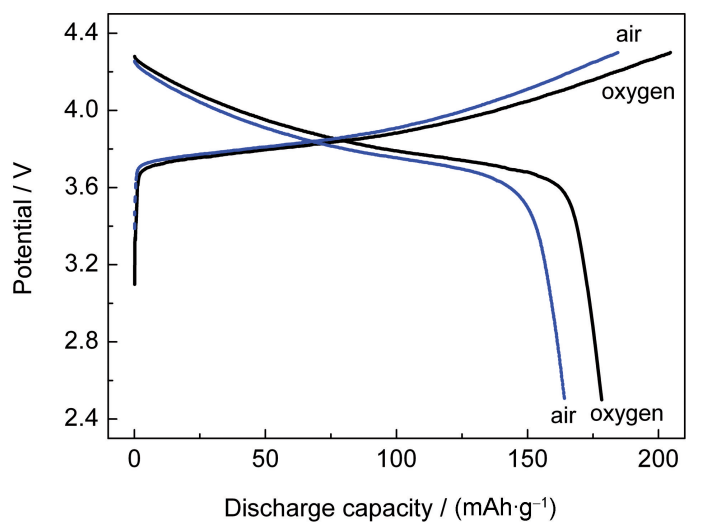

图 5 不同焙烧气氛下合成的 $\mathrm{LiNi}_{0.5} \mathrm{Mn}_{0.5} \mathrm{O}_{2}$ 的首次放电 容量图

Fig.5 The first discharge capacity of $\mathrm{LiNi}_{0.5} \mathrm{Mn}_{0.5} \mathrm{O}_{2}$ synthesized under different calcination atmospheres
性, 更加完美的层状结构和较小的 $\mathrm{Li} / \mathrm{Ni}$ 混排率. 5,23

图 6 是不同焙烧气氛下合成的 $\mathrm{LiNi}_{0.5} \mathrm{Mn}_{0.5} \mathrm{O}_{2}$ 的 循环图, 充放电电压区间为 2.5-4.3 V, 充放电倍率 为 $0.1 C$. 从图中可以看出氧气气氛下合成的材料显 示出良好的循环性能. 经过 50 圈循环以后, 放电容 量为 $165 \mathrm{mAh} \cdot \mathrm{g}^{-1}$, 容量保持率为 $92.7 \%$. 空气气氛

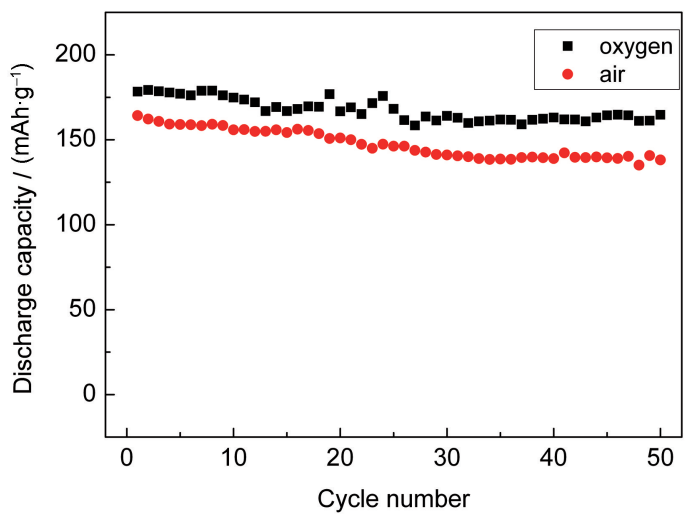

图 6 不同气氛下合成的 $\mathrm{LiNi}_{0.5} \mathrm{Mn}_{0.5} \mathrm{O}_{2}$ 的循环图

Fig.6 Cycle performance of $\mathrm{LiNi}_{0.5} \mathrm{Mn}_{0.5} \mathrm{O}_{2}$ calcined under different atmospheres discharge rate: $0.1 C$ 


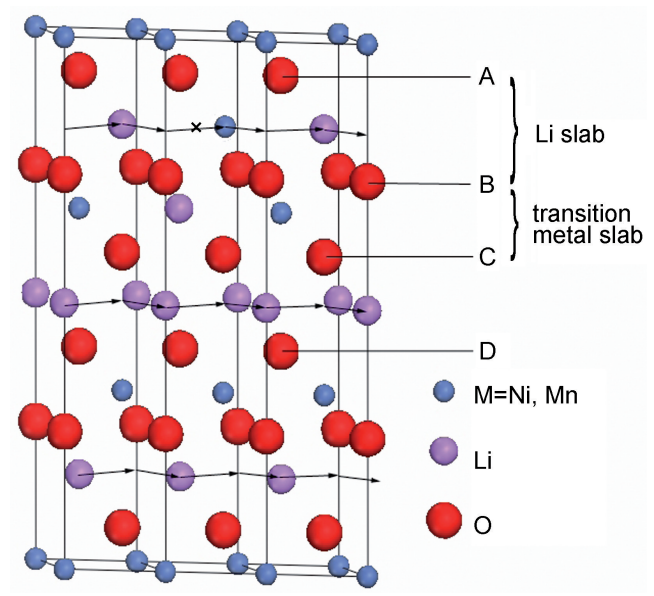

图 7 层状 $\mathrm{LiNi}_{0.5} \mathrm{Mn}_{0.5} \mathrm{O}_{2}$ 材料晶体结构中 $\mathrm{Li} / \mathrm{Ni}$ 混排示意图 Fig.7 Schematic lattice structure of layered $\mathrm{LiNi}_{0.5} \mathrm{Mn}_{0.5} \mathrm{O}_{2}$ materials with $\mathrm{Li} / \mathrm{Ni}$ disorder

下合成的材料经过 50 圈循环以后, 放电容量为 137 $\mathrm{mAh} \cdot \mathrm{g}^{-1}$, 容量保持率为 $83.5 \%$. 这可能归因于在氧 气气氛下合成的 $\mathrm{LiNi}_{0.5} \mathrm{Mn}_{0.5} \mathrm{O}_{2}$ 具有更好的结晶性, 更加完美的层状结构和较小的 $\mathrm{Li} / \mathrm{Ni}$ 混排率.

\section{3 晶体中 $\mathrm{Li} / \mathrm{Ni}$ 混排率与电化学性能的关系}

图 7 是层状 $\mathrm{LiNi}_{0.5} \mathrm{Mn}_{0.5} \mathrm{O}_{2}$ 材料晶体结构中 $\mathrm{Li} / \mathrm{Ni}$ 混排示意图. 晶体结构中的 $\mathrm{Li} / \mathrm{Ni}$ 混排会严重影响到 材料的电化学性能. ${ }^{5}$ 当 $\mathrm{Ni}$ 离子混排进入锂离子层占 据锂离子位置时, 会阻断锂离子传输的路径, 减少在 充放电循环中可以参与的锂离子数量. 由于容量是 与在充放电过程中可以嵌入和脱出的锂离子的数量 有关系的, 因此可传递的锂离子数量的降低将导致 材料的容量降低. 这一结论与电化学测试结论相符. 锂离子传递的难易程度与锂离子传输的空间有很大 关系. 锂离子层间距越小, 锂离子的传递越困难. ${ }^{5}$ 当 锂离子混排进入过渡金属层时, 由于锂离子的半 径 $\left(r_{\mathrm{Li}^{+}}=0.076 \mathrm{~nm}\right)$ 大于过渡金属离子的半径 $\left(r_{\mathrm{Ni}^{+}}=\right.$ $0.068 \mathrm{~nm}, r_{\mathrm{Mn}^{+}}=0.053 \mathrm{~nm}^{23}$ ), 由于空间效应和正电 荷之间的互相排斥, 过渡金属层会因此而膨胀, 层间 距扩大, 相邻的锂离子层就会因为过渡金属层的膨 胀而受到挤压, 导致锂离子层间距变小, 较小的锂离 子层间距会降低锂离子的扩散系数. 为了验证这一 理论推算, 我们对材料做了电化学阻抗测试.

\section{4 电化学阻抗测试结果分析}

图 8 是不同焙烧气氛下合成的 $\mathrm{LiNi}_{0.5} \mathrm{Mn}_{0.5} \mathrm{O}_{2}$ 的 电化学阻抗谱(EIS)图. 每个EIS 图都是由一个半圆 和一条直线组成的. 锂离子扩散系数 $D\left(\mathrm{~cm}^{2} \cdot \mathrm{s}^{-1}\right)$ 可 以通过 EIS 图用公式(1)计算出来. ${ }^{24-26}$

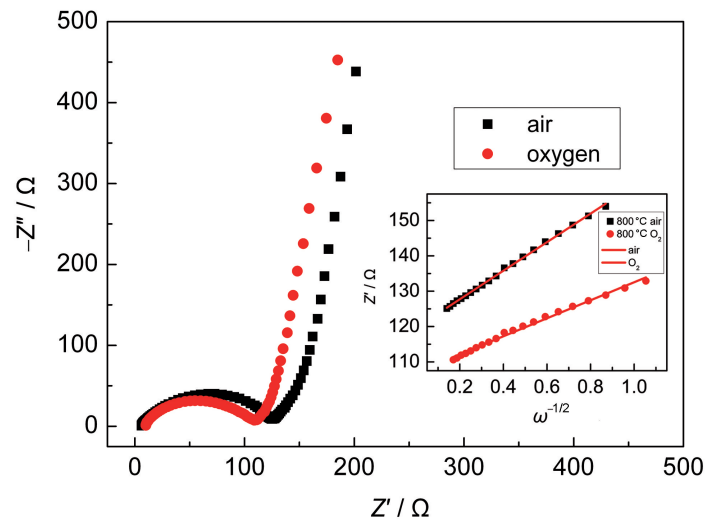

图 8 不同焙烧气氛下合成的 $\mathrm{LiNi}_{0.5} \mathrm{Mn}_{0.5} \mathrm{O}_{2}$ 的 $\mathrm{EIS}$ 谱图 Fig.8 EIS spectra of $\mathrm{LiNi}_{0.5} \mathrm{Mn}_{0.5} \mathrm{O}_{2}$ synthesized under different calcination atmospheres

$Z^{\prime}$ is the real part of Nyquist impedance, $Z^{\prime \prime}$ is the imaginary part of Nyquist impedance, $\omega$ is the frequency in the electrochemical impedance spectroscopy test.

$$
D=\frac{R^{2} T^{2}}{2 A^{2} n^{4} F^{4} C^{2} \sigma^{2}}
$$

式中, $A$ 是极片的几何面积, $n$ 是化学反应中传递的 电子数, $F$ 是法拉第常数, $C$ 是锂离子在极片中的浓 度, $\sigma$ 是直线 $Z^{\prime}-\omega^{-1 / 2}$ 的斜率, $R$ 是气体常数, $T$ 是实验 时的开尔文温度. 在此公式中, $F$ 和 $R$ 为常数, $n, A, C$ 在两个样品测试实验中保持一致, 唯一影响计算结 果的是 $\sigma$. 计算结果显示在氧气气氛下合成材料的锂 离子扩散系数为 $3.54 \times 10^{-13} \mathrm{~cm}^{2} \cdot \mathrm{s}^{-1}$, 大于空气气氛下 合成材料的锂离子扩散系数 $\left(1.41 \times 10^{-13} \mathrm{~cm}^{2} \cdot \mathrm{s}^{-1}\right)$. 氧 气气氛下较大的锂离子扩散系数可能归因于晶体结 构中较小的 $\mathrm{Li} / \mathrm{Ni}$ 混排率. 与理论模型推算结构相 符, 说明较大的 $\mathrm{Li} / \mathrm{Ni}$ 混排率会对材料的电化学性能 带来负面的影响.

\section{4 结 论}

用固相法分别在空气和氧气的气氛下合成了纯 相的 $\mathrm{LiNi}_{0.5} \mathrm{Mn}_{0.5} \mathrm{O}_{2}$. 经过表征和电化学测试, 显示氧 气气氛下焙烧出的材料电化学性能明显优于空气气 氛下合成的材料. 氧气气氛下合成的材料首次放电 容量达到 $178 \mathrm{mAh} \cdot \mathrm{g}^{-1}$, 充放电循环 50 圈后容量为 $165 \mathrm{mAh} \cdot \mathrm{g}^{-1}$, 容量保持率为 $92.7 \%$. 这可能归因于 氧气气氛下合成的样品具有良好的层状结构, 好的 晶体结晶性, 较大的锂离子扩散系数.

\section{References}

(1) Scrosati, B. Electrochim. Acta 2000, 45, 2461.

(2) Ohzuku, T.; Makimura, Y. Chem. Lett. 2001, 8, 744. 
(3) Sun, Y. K.; Bae, Y. C.; Myung, S. T. J. Appl. Electrochem. 2005, $35,151$.

(4) Zhou, Y. K.; Li, H. L. J. Mater. Chem. 2002, 12, 681.

(5) Kang, K. S.; Meng, Y. S.; Breger, J.; Grey, C. P.; Ceder, G. Science 2006, 311, 977.

(6) Ariyoshi, K.; Tomohiro, I.; Ohzuku, T. J. Phys. Chem. Solids 2008, 69, 1238.

(7) Kang, S. H.; Park, S. H.; Johnson, C. S.; Amine, K. J. Electrochem. Soc. 2007, 154, A268.

(8) Abdel, G. A.; Zaghib, K.; Gendron. F.; Mauger. A.; Julien, C. M. Electrochim. Acta 2007, 52, 4092.

(9) Yabuuchi, N.; Kumar, S.; Li, H. H.; Kim, Y. T. J. Electrochem. Soc. 2007, 154, A566

(10) Islam, M. S.; Davies, R. A.; Gale, J. D. Chem. Mater. 2003, 22, 4280 .

(11) Wu, Q.; Lu, X. Y.; Yan, M. M.; Jiang, Z. Y. Electrochem. Commun. 2003, 10, 878.

(12) Sun, Y. K.; Myung, S. T.; Kim, M. H.; Prakash, J.; Amine, K. J. Am. Chem. Soc. 2005, 38, 13411.

(13) Hwang, B. J.; Yu, T. H.; Cheng, M. Y., Santhanam, R. J. Mater. Chem. 2009, 19, 4536.

(14) Hinuma, Y.; Meng, Y. S.; Kang, K.; Ceder, G. Chem. Mater.
2007, 19, 1790 .

(15) Yoshio, M.; Todorov, Y.; Yamato, K.; Noguchi, H.; Itoh, M. J.; Okada, M. T. J. Power Sources 1998, 74, 46.

(16) Park, S. H.; Sun, Y. K. Electrochim. Acta 2004, 50, 431.

(17) Ammundsen, B.; Paulsen, J. Adv. Mater. 2001, 13, 943.

(18) Makimura, Y.; Ohzuku, T. J. Power Sources 2003, 119-121, 156.

(19) Lu, Z. H.; Beaulieu, L. Y.; Donaberger, R. A.; Thomas, C. L.; Dahn, J. R. J. Electrochem. Soc. 2002, 149, 778.

(20) Whittingham, M. S. Chem. Rev. 2004, 104, 4271.

(21) Lu, Z. H.; MacNeil, D. D.; Dahn, J. R. Electrochemical and Solid-State Letters 2001, 4, 200.

(22) Zhong, H.; Xu, H. Acta. Chim. Sin. 2004, 62, 1123. [钟 辉, 许 惠. 化学学报, 2004, 62, 1123.]

(23) Lian, F.; Axmann, P.; Stinner, C.; Liu, Q. G.; Wohlfahrt, M. M. J. Appl. Electrochem. 2008, 38, 613.

(24) Liu, J. L.; Jiang, R. R.; Wang, X. Y.; Huang, T.; Yu, A. S. J. Power Sources 2009, 194, 536.

(25) Gao, F.; Tang, Z. Y. Electrochim. Acta 2008, 53, 5071.

(26) Liao, X. Z.; Ma, Z. F.; Qiang, G.; He, Y. S.; Li, P.; Zeng, L. J. Electrochem. Commun. 2008, 10, 691. 